\title{
Article \\ Duty Cycle Control Method Considering Buffer Occupancy for IEEE 802.15.4-Compliant Heterogeneous Wireless Sensor Network
}

\author{
Kohei Tomita ${ }^{1}$ and Nobuyoshi Komuro ${ }^{2, *(D)}$ \\ 1 Graduate School of Science and Engineering, Chiba University, Chiba-shi, Chiba 263-8522, Japan; \\ tomihey@chiba-u.jp \\ 2 Institute of Media and Information Technologies, Chiba University, Chiba-shi, Chiba 263-8522, Japan \\ * Correspondence: kmr@faculty.chiba-u.jp; Tel.: +81-43-290-3544
}

check for updates

Citation: Tomita, K.; Komuro, N. Duty Cycle Control Method Considering Buffer Occupancy for IEEE 802.15.4-Compliant Heterogeneous Wireless Sensor Network. Appl. Sci. 2021, 11, 1362. https://doi.org/10.3390/app11041362

Received: 24 December 2020 Accepted: 1 February 2021 Published: 3 February 2021

Publisher's Note: MDPI stays neutral with regard to jurisdictional clai$\mathrm{ms}$ in published maps and institutional affiliations.

Copyright: (C) 2021 by the authors. Licensee MDPI, Basel, Switzerland. This article is an open access article distributed under the terms and conditions of the Creative Commons Attribution (CC BY) license (https:// creativecommons.org/licenses/by/ $4.0 /)$.

\begin{abstract}
This paper proposes a Duty-Cycle (DC) control method in order to improve the Packet Delivery Ratio (PDR) for IEEE 802.15.4-compliant heterogeneous Wireless Sensor Networks (WSNs). The proposed method controls the DC so that the buffer occupancy of sensor nodes is less than 1 and assigns DC to each sub-network (sub-network means a network consisting of a router node and its subordinate nodes). In order to use the appropriate DC of each sub-network to obtain the high PDR, this paper gives analytical expressions of the buffer occupancy. The simulation results show that the proposed method achieves a reasonable delay and energy consumption while maintaining high PDR.
\end{abstract}

Keywords: IEEE 802.15.4; duty-cycle; active period control; heterogeneous sensor network; tree topology

\section{Introduction}

Ad-hoc sensor networks have been paid attention in various applications, such as environment monitoring and smart home [1-16]. Today, heterogeneous Wireless Sensor Networks (WSNs) also gain enormous attention due to the diversification of applications, such as medical applications and environmental monitoring systems $[17,18]$. In heterogeneous WSNs, various components are heterogeneous, such as sensor nodes' traffic sources (low/middle/high) and network topology (star/tree). Therefore, it is difficult to control heterogeneous WSNs nodes' operations because of their complexities.

The IEEE 802.15.4 is the standard of WSN. The devices communicate with superframe structure in the IEEE 802.15.4 standard [19]. In the superframe structure, the communication of each node is managed by the active and sleep cycles (Duty-Cycle). The energy consumption of WSN nodes are saved by controlling DC. The Quality of Service (QoS), such as Packet Delivery Ratio (PDR) and delay, is controlled by tuning the MAC (Medium Access Control) parameters [18,20-22].

Z. Xiao et al. proposed a scheduling protocol for star topology WSNs [20]. The protocol proposed in Reference [20] controls active and sleep periods taking into account the buffer occupancy of sensor nodes. However, the protocol proposed in Reference [20] is not suitable for controlling heterogeneous WSNs nodes' operations since it is proposed under the assumption of the homogeneous networks.

A. Karalis et al. proposed a beacon signal avoidance protocol for homogeneous tree topology WSNs in order to achieve high PDR and low energy consumption [22]. Although the collision avoidance protocol proposed in Reference [22] can avoid beacon signal collisions, it does not guarantee collision avoidance of outgoing packets of sensor nodes.

The Tele-Medicine Protocol (TMP) [18] is an IEEE 802.15.4-based MAC protocol for heterogeneous WSNs. The TMP tunes DC and MAC layer parameters to meet the QoS requirements in the medical field. The TMP controls the active-period length of a superframe 
to be shorter in order to reduce energy consumption. However, for high data rate nodes, the shorter the active period, the more packets are lost, and the lower the PDR [23-26].

This paper proposes a duty-cycle control method in order to improve the PDR for IEEE 802.15.4-compliant heterogeneous WSNs. The proposed method tunes the DC so that the buffer occupancy of sensor nodes is less than one and assigns DC to each sub-network (sub-network means a network consisting of a router node and its subordinate nodes). The appropriate DC of each sub-network can be used to obtain the high PDR; thus, this paper gives analytical expressions of the buffer occupancy. The proposed method can control DC heterogeneous star and/or tree-topology wireless sensor networks. Simulation results show the effectiveness of the proposed method.

\section{Related Works}

\subsection{IEEE 802.15.4}

In the IEEE 802.15.4 beacon enable mode, the devices communicate using the superframe structure. The superframe consists of three parts: the beacon frame, active period, and inactive period, as shown in Figure 1. Nodes send and receive packets during only the active period. The active period is divided into CAP (Contention Access Period) and CFP (Contention Free Period). The devices communicate using CSMA/CA in the CAP. The proposed method assumes to use only CAP.

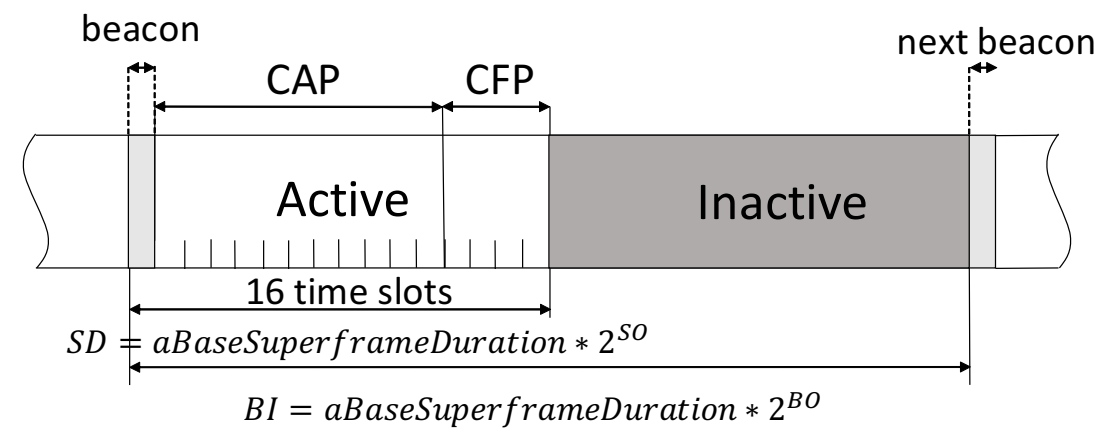

Figure 1. The superframe structure of IEEE 802.15.4.

Figure 2 shows the flow chart of IEEE 802.15.4 Clear Channel Access (CCA). MacCSMABackoffs is the number of retransmissions. Contention windows $(\mathrm{CW})$ are used to communicate fairly. Each node checks whether the channel is idle before sending packets.

Beacon Interval (BI) means the time interval between two superframes. The length of $\mathrm{BI}$ is determined by the parameter $\mathrm{BO}$ (Beacon Order), which is expressed by

$$
B I=\text { aBaseSuperframeDuration } \times 2^{B O}(\mathrm{~ms}),
$$

where aBaseSuperframeDuration is the basic length of the superframe and consists of 16 time slots, and one time slot length is $0.96 \mathrm{~ms}$. So, the aBaseSuperframeDuration is $15.36 \mathrm{~ms}$. Superframe Duration (SD) means the length of the active period. The length of SD is determined by SO (Superframe Order), which is expressed by

$$
S D=\text { aBaseSuperframeDuration } \times 2^{S O}(m s) .
$$

From Figure 1, $\mathrm{BO}$ and $\mathrm{SO}$ satisfy $\mathrm{SO} \leq \mathrm{BO}$. The ratio of the length of the active period to that of the superframe is called DC. DC is expressed by

$$
D C=\frac{2^{S O}}{2^{B O}} .
$$




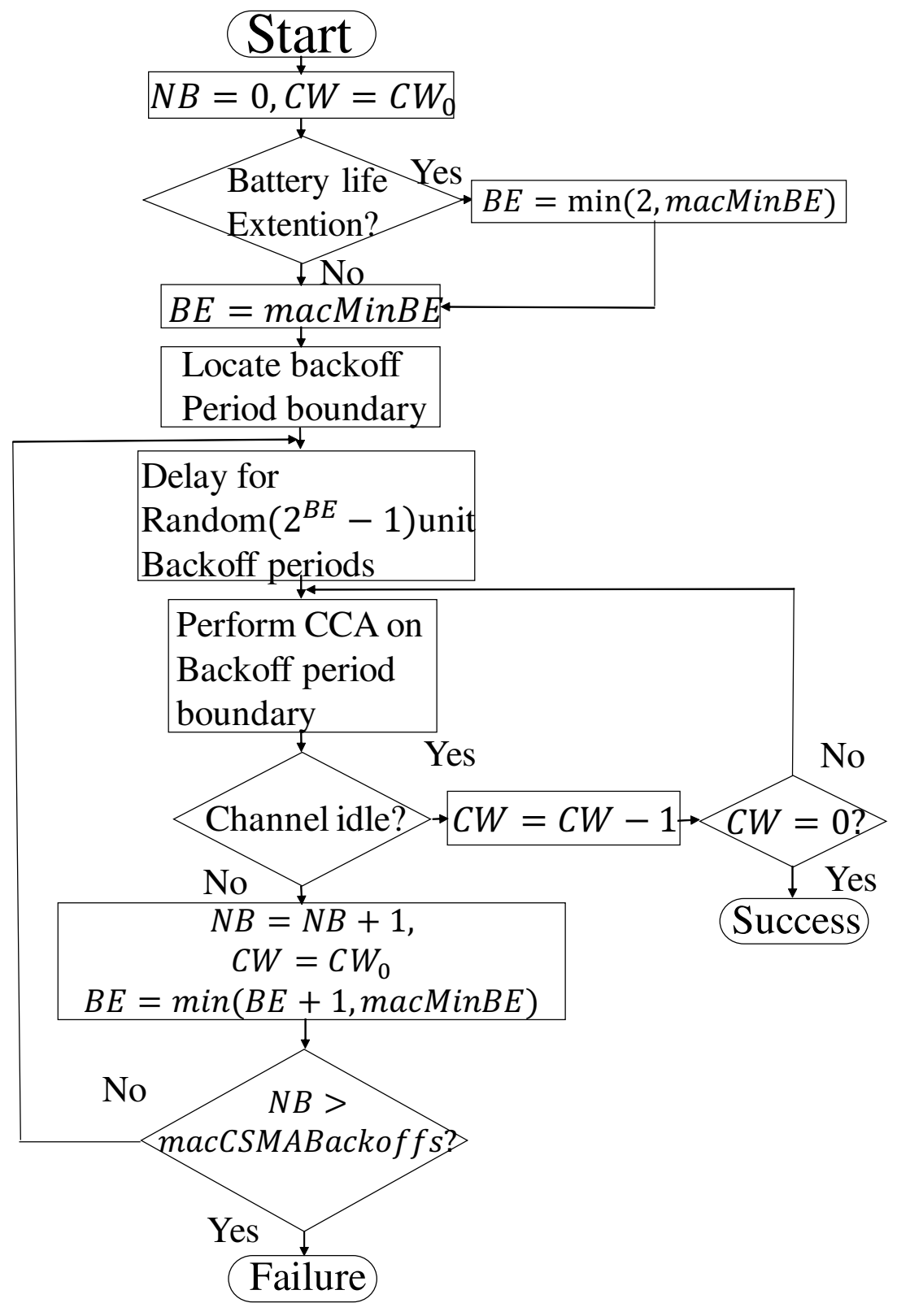

Figure 2. Flow chart of IEEE 802.15.4 packet transmission.

\subsection{TMP (Tele-Medicine Protocol)}

The TMP tunes DC for heterogeneous WSNs. In the TMP, DC is controlled to reduce energy consumption while meeting the allowable delay. The energy consumption increases as the active period become longer. Therefore, the TMP sets DC as small as possible to minimize energy consumption while guaranteeing QoS.

\section{Proposed Method}

This paper proposes a DC control method to achieve high PDR for IEEE 802.15.4compliant heterogeneous star and/or tree topology WSNs. Figure 3 shows the example of a tree topology heterogeneous WSNs. In homogeneous WSN, communication qualities, such as PDR and energy consumption, can be improved by setting the same DC for all sensor nodes. On the other hand, in heterogeneous WSNs where data rates of sensor nodes are different, even if the same DC control is applied to all sensor nodes, the high communication quality may not be achieved because of the different characteristics of 
the network nodes. In order to solve this problem, the proposed method allocates an appropriate DC for each sub-network (sub-network means a network consisting of a router node and its subordinate nodes). DC is controlled in consideration of the buffer occupancy of nodes.

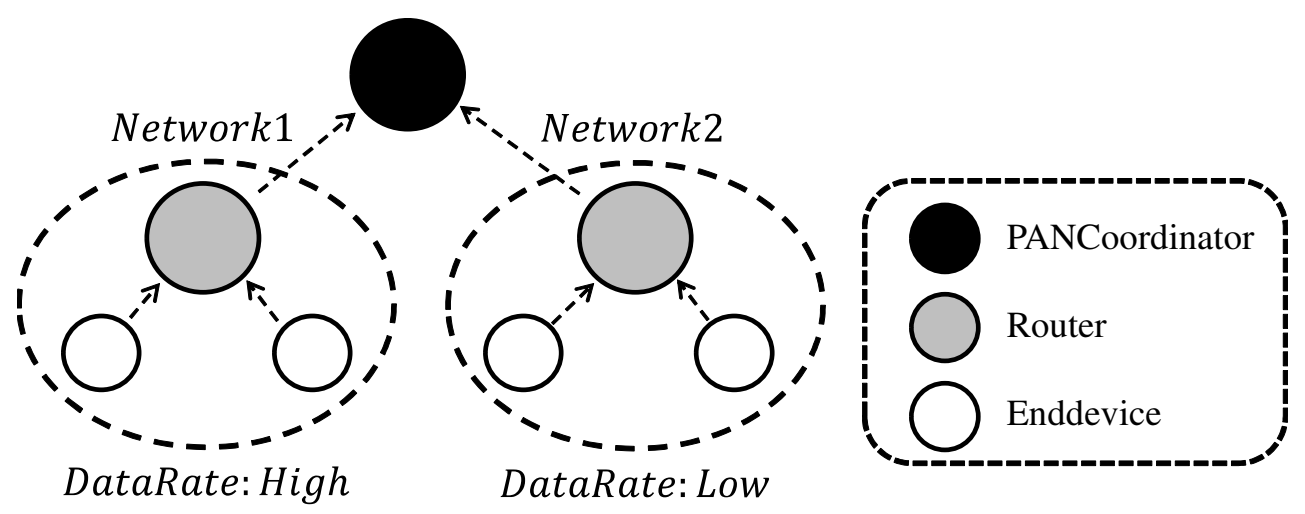

Figure 3. Example of a tree topology heterogeneous Wireless Sensor Networks (WSNs).

This paper assumes that each router node does not generate its own packet. In this section, we also assume that there are no transmission failures. Each router node generates a packet when relaying packets from the end devices. We assume that the number of generating packets of router node $i$ in one superframe duration $\lambda_{i}$ is given by

$$
\lambda_{i}=\frac{\text { DataRate }_{i}}{8 \times P} \times B I,
$$

where DataRate $e_{i}$ is the total data rate of end devices which belong to router node $i$, and $P$ is a packet payload size (byte).

Assuming that there are no transmission failures, the data rate of router node $i$ is given by

$$
\text { DataRate }_{i}=\sum_{j=1}^{N_{i}} \text { DataRate }_{j},
$$

where $N_{i}$ is the number of end devices which belong to router node $i$. Assuming that router node $i$ transmits $n_{i}$ packets during aBaseSuperframeDuration, the total number of transmitted packets during one active period, which indicates the service rate of node $i$, $\mu_{i}$, is

$$
\mu_{i}=n_{i} \times 2^{S O} .
$$

Assuming no contention, $n_{i}$ is given by

$$
n_{i}=\frac{\text { aBaseSuperframeDuration }- \text { Beaconframe }}{\text { maxDelay }},
$$

where maxDelay is given by Equation (8), and expressed as

$$
\begin{array}{r}
\text { maxDelay }=\text { macCSMABackoff } *\left(T_{\text {Backoff }}+T_{C C A}\right) \\
+T_{P k t T x}+T_{A C K}+T_{S I F S}+T_{T A}
\end{array}
$$

where macCSMABackoff is the maximum number of carrier sensing trials, $T_{\text {Backoff }}$ and $T_{C C A}$ are time to backoff and carrier sense, respectively. $T_{P k t T x}$ expresses the time to transmit a packet. When the data transfer speed is 250 (Kbps),

$$
T_{\text {PktTX }}=\frac{\text { PacketSize }(\text { byte })}{250(\text { Kbps })} .
$$


In Equation (8), $T_{A C K}$ is the time to transmit an ACK frame, $T_{S I F S}$ is short inter frame space, and $T_{T A}$ is the waiting time for the next transmission.

From Equations (4) and (6), the buffer occupancy of router node $i \rho_{i}$ is expressed as the ratio of the number of generating packets of router node $i$ in one superframe duration, $\lambda_{i}$, to the service rate of node $i, \mu_{i}$. $\rho_{i}$ is given by

$$
\begin{aligned}
\rho_{i} & =\frac{\lambda_{i}}{\mu_{i}} \\
& =\frac{\frac{\text { DataRate }_{i}}{8 \times P} \times \text { aBaseSuperframeDuration } \times 2^{B O}}{n_{i} \times 2^{S O}} .
\end{aligned}
$$

For avoiding buffer overflow, DC should be controlled so that $\rho_{i}$ is less than 1 , as shown in Figure 4. From Equations (4)-(10), DC should satisfy:

$$
D C>\frac{\text { DataRate }_{i}}{8 \times P} \times \frac{\text { aBaseSuperframeDuration }}{n_{i}} .
$$

$\lambda$ (Number of packet generated during Superframe)

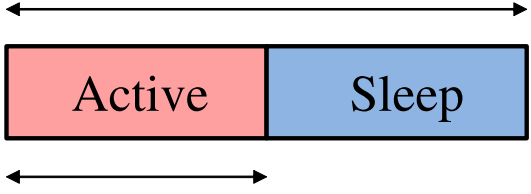

$\mu$ (Number of packet processed during active period)

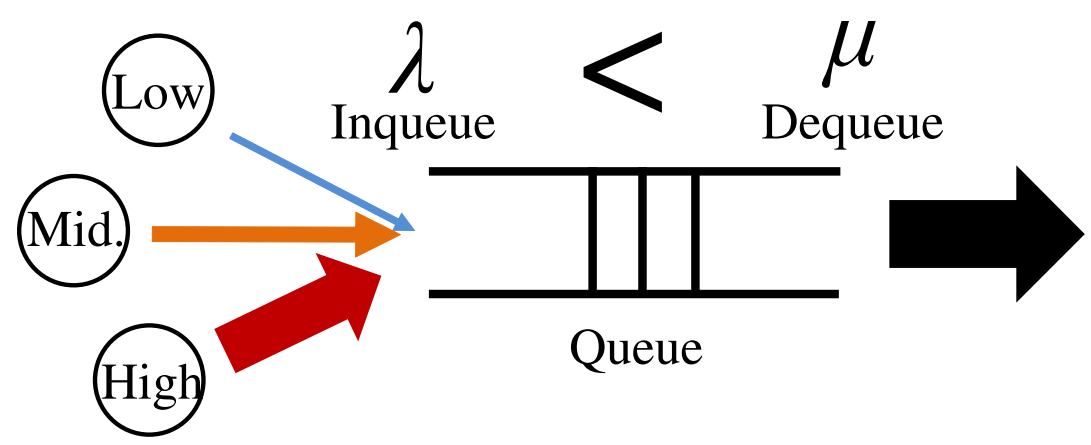

Figure 4. Buffer model in the proposed method.

\section{Simulation Results}

The proposed method is evaluated by Network Simulator 2 (NS2) [27]. Table 1 shows the simulation parameters. Simulation is conducted by using a frequency band of $2.4 \mathrm{GHz}$. In this simulation, the energy consumption parameters of CC2420 [28] are used; Tx energy is $31.25 \mathrm{~mW}$, Rx energy is $35.28 \mathrm{~mW}$, Idle energy is $0.712 \mathrm{~mW}$, and Sleep energy is $0.144 \mathrm{~mW}$. In addition, this paper uses IEEE 802.15.4 default MAC parameters [19].

This study assumes that the packet transmissions are failed due to collisions caused by concurrent transmissions.

This study does not assume transmission failures due to physical layer conditions. 
Table 1. Simulation parameters.

\begin{tabular}{cc}
\hline Parameters & Value \\
\hline Frequency band & $2.4(\mathrm{GHz})$ \\
Packet length & $100(\mathrm{byte})$ \\
Simulation time & $10-1000(\mathrm{~s})$ \\
Tx energy & $31.25(\mathrm{~mW})$ \\
Rx energy & $35.28(\mathrm{~mW})$ \\
Idle energy & $0.712(\mathrm{~mW})$ \\
Sleep energy & $0.144(\mathrm{~mW})$ \\
macMaxBE & 5 \\
macMinBE & 3 \\
macCSMABackoff & 4 \\
macMaxFrameRetries & 3 \\
\hline
\end{tabular}

\subsection{Star Topology WSN}

Figures 5 and 6 show the PDRs of the TMP method [18] and the proposed method in the star topology WSN, respectively. The data rate of each node is shown in Table 2. In the TMP method, the PDR of end devices decrease as the number of nodes increases. This is because the TMP method does not adequately control DC for high data rate nodes. On the other hand, in the proposed method, the PDR of end devices do not decrease so much. It is seen from this result that the proposed method can cope with the increase in the data rate.

Table 2. Data rate of each node (bps).

\begin{tabular}{ccccccccc}
\hline NodeID & N1 & N2 & N3 & N4 & N5 & N6 & N7 & N8 \\
\hline DataRate & 444 & 500 & 570 & 667 & 800 & $1 \mathrm{~K}$ & $1.3 \mathrm{~K}$ & $2 \mathrm{~K}$ \\
\hline
\end{tabular}

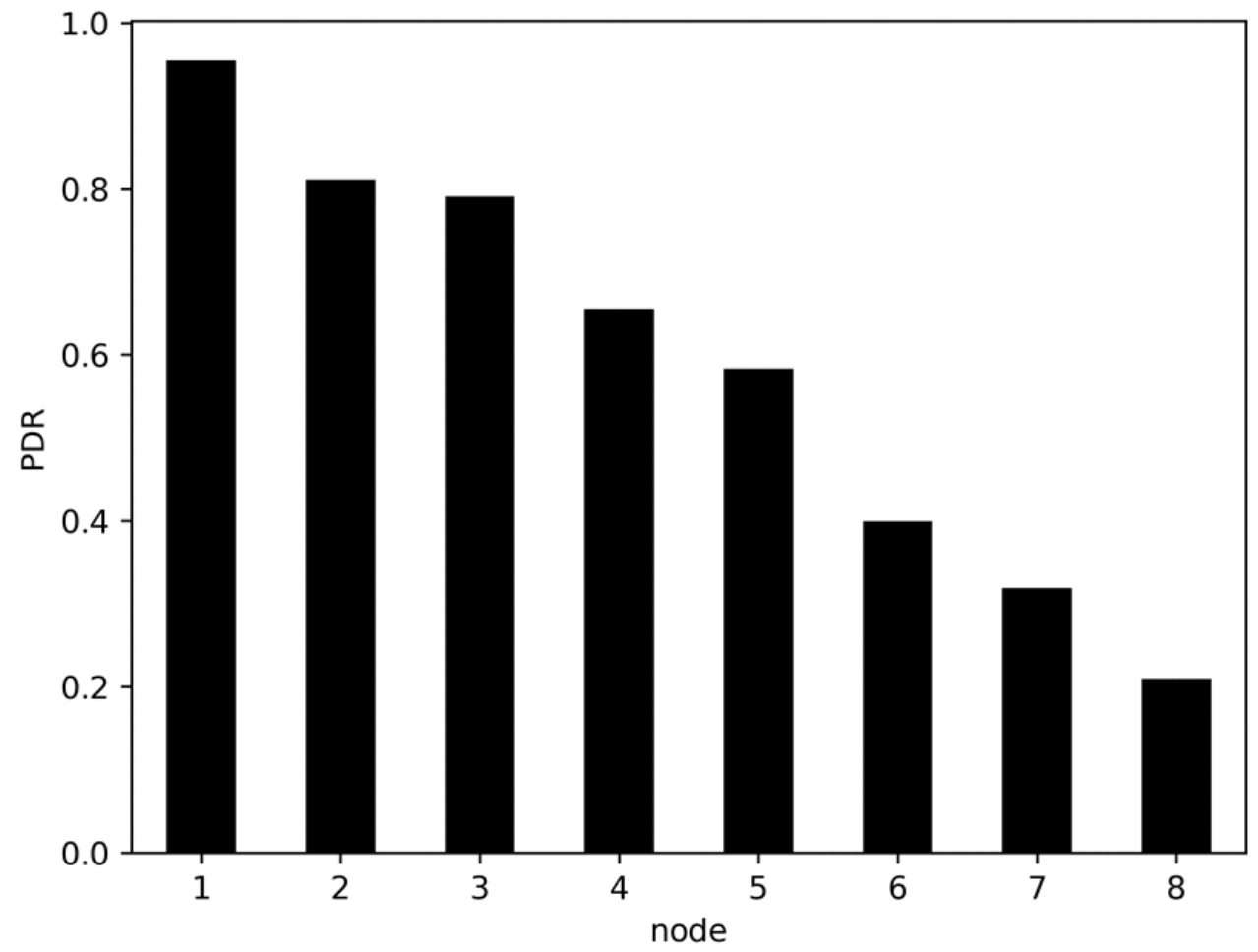

Figure 5. Packet Delivery Ratio (PDR) of each node in Tele-Medicine Protocol (TMP) method. 


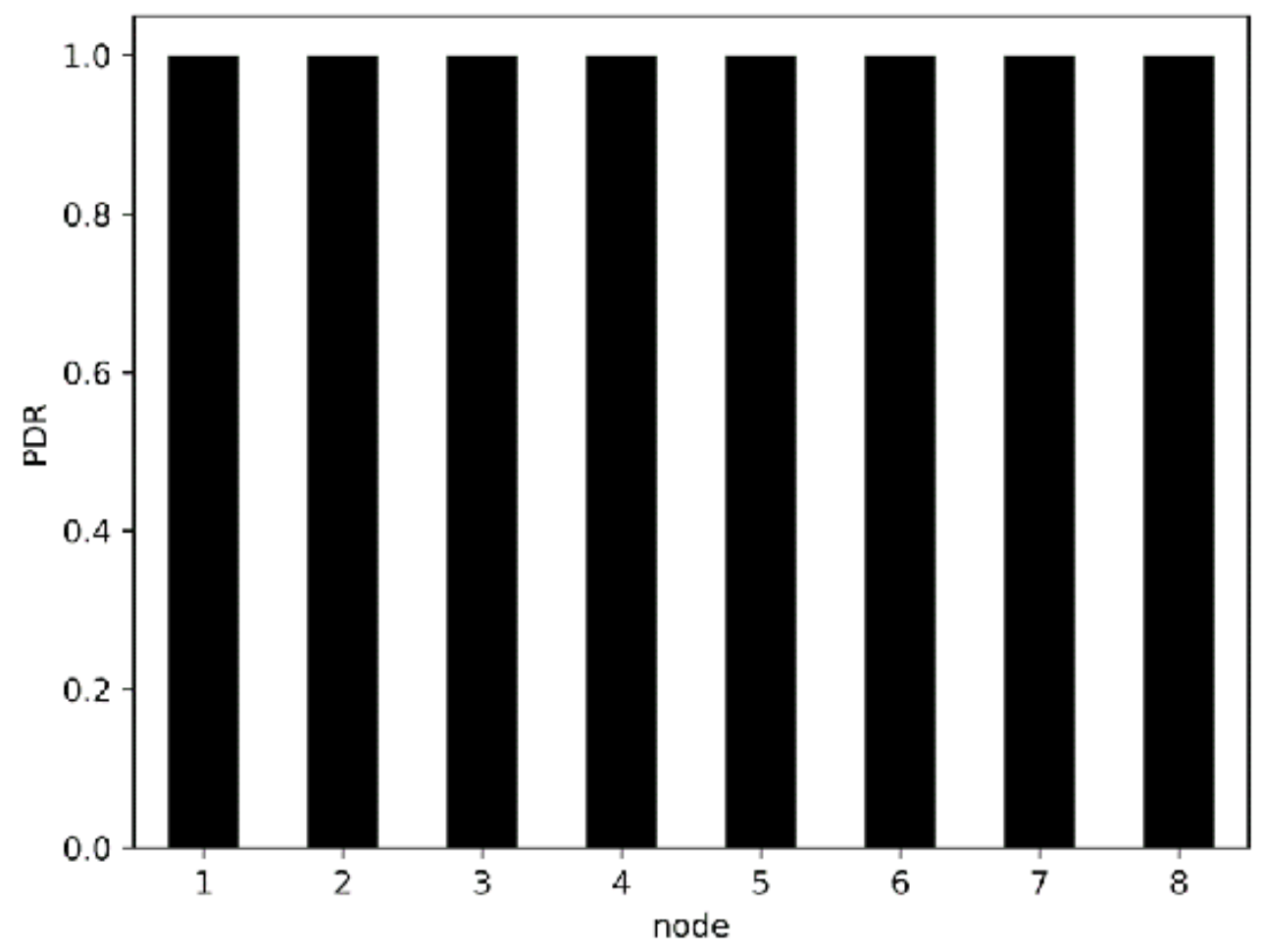

Figure 6. PDR of each node in the proposed method.

\subsection{Tree Topology WSNs}

Figure 7 shows the tree topology WSNs used in this simulation. Nodes 1 and 2 are route nodes. Networks 1 and 2 are composed of router node 1 and its end devices and router node 2 and its end devices, respectively. Each router node does not generate its own packet. Each router node generates a packet when relaying packets from the end devices. This study compares the proposed method, the DC control method considering buffer occupancy for 802.15.4-compliant homogeneous WSN, and the TMP method. We evaluate the proposed method under three scenarios. Table 3 shows the DC for each method. The proposed method uses three DC patterns since the proposed method tunes the DC according to the data rate of the subordinate nodes. Table 4 shows data rate of each node. In these tables, $D C_{i}$ and DataRate ${ }_{i}$ are DC and data rate of router node $i$, respectively.

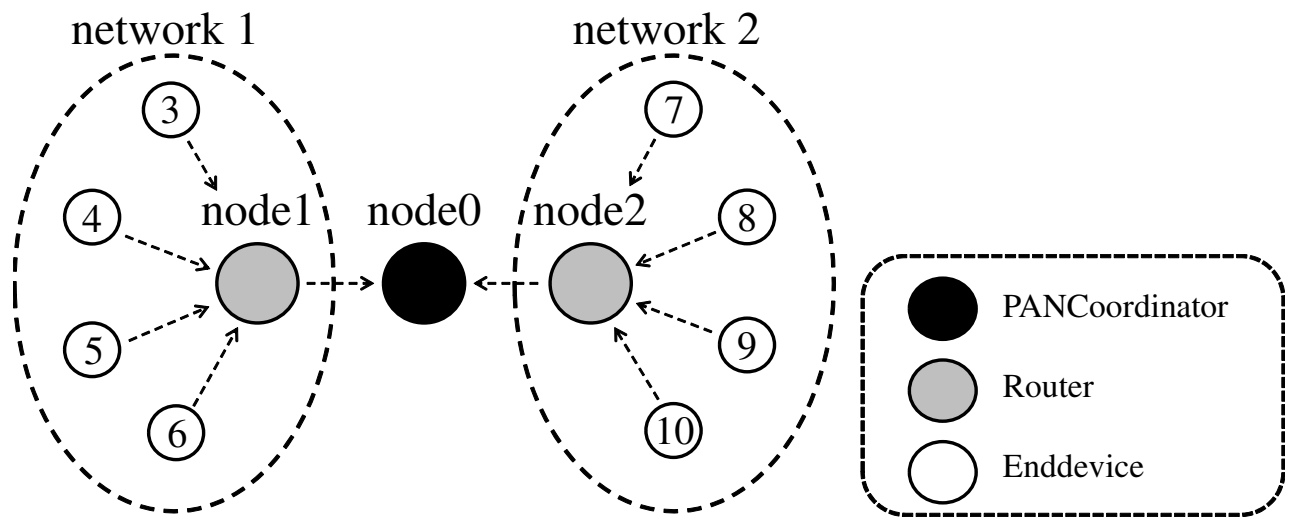

Figure 7. Network topology in the simulation. 
Table 3. Duty-Cycle (DC) for each method.

\begin{tabular}{ccc}
\hline Scenario & DC1 & DC2 \\
\hline 802.15.4 & 1.0 & 1.0 \\
TMP & 0.125 & 0.125 \\
Prop-scenario1 & 0.125 & 1.0 \\
Prop-scenario2 & 0.125 & 0.5 \\
Prop-scenario3 & 0.5 & 1.0 \\
\hline
\end{tabular}

Table 4. Data rate of each node (bps).

\begin{tabular}{ccccccccc}
\hline Scenario & N3 & N4 & N5 & N6 & N7 & N8 & N9 & N10 \\
\hline Scenario-1 & 200 & 200 & 400 & 800 & $1.6 \mathrm{~K}$ & $2 \mathrm{~K}$ & $2.7 \mathrm{~K}$ & $4 \mathrm{~K}$ \\
Scenario-2 & 200 & 200 & 400 & 800 & 200 & 400 & 800 & $2 \mathrm{~K}$ \\
Scenario-3 & 200 & 400 & 800 & $2 \mathrm{~K}$ & $1.6 \mathrm{~K}$ & $2 \mathrm{~K}$ & $2.7 \mathrm{~K}$ & $4 \mathrm{~K}$ \\
\hline
\end{tabular}

\subsubsection{PDR Evaluation}

Figures 8 and 9 show the number of packets in the buffer of a sensor node with and without appropriate DC control, respectively. In Figure 9, the number of packets in the buffer increases in time. On the other hand, in Figure 8, the number of packets in the buffer does not increase because the generated packets are processed in the active period. It is seen from Figures 8 and 9 that the appropriate DC control can avoid packet accumulation in buffer.

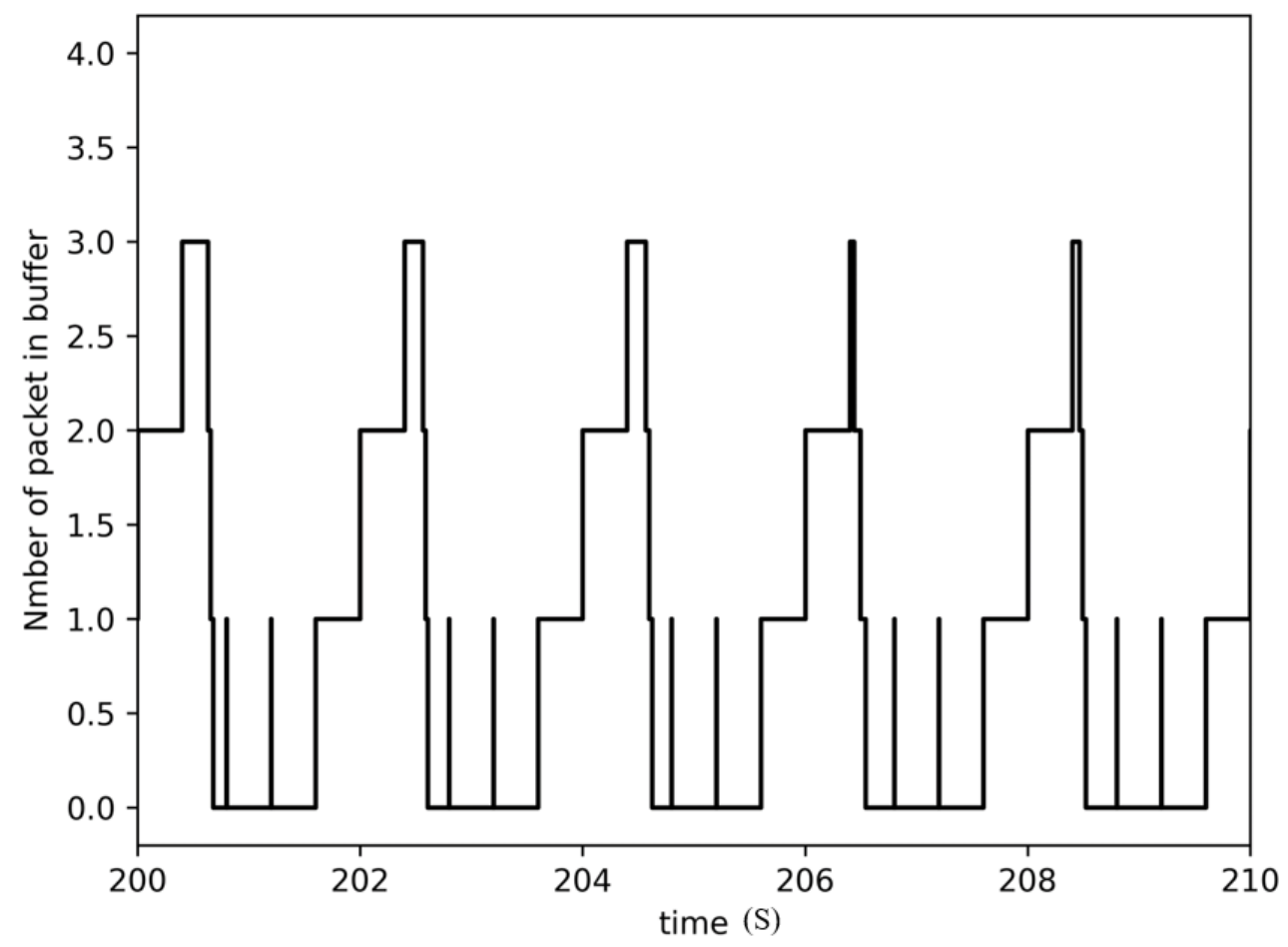

Figure 8. Number of packets in buffer with appropriate DC control. 


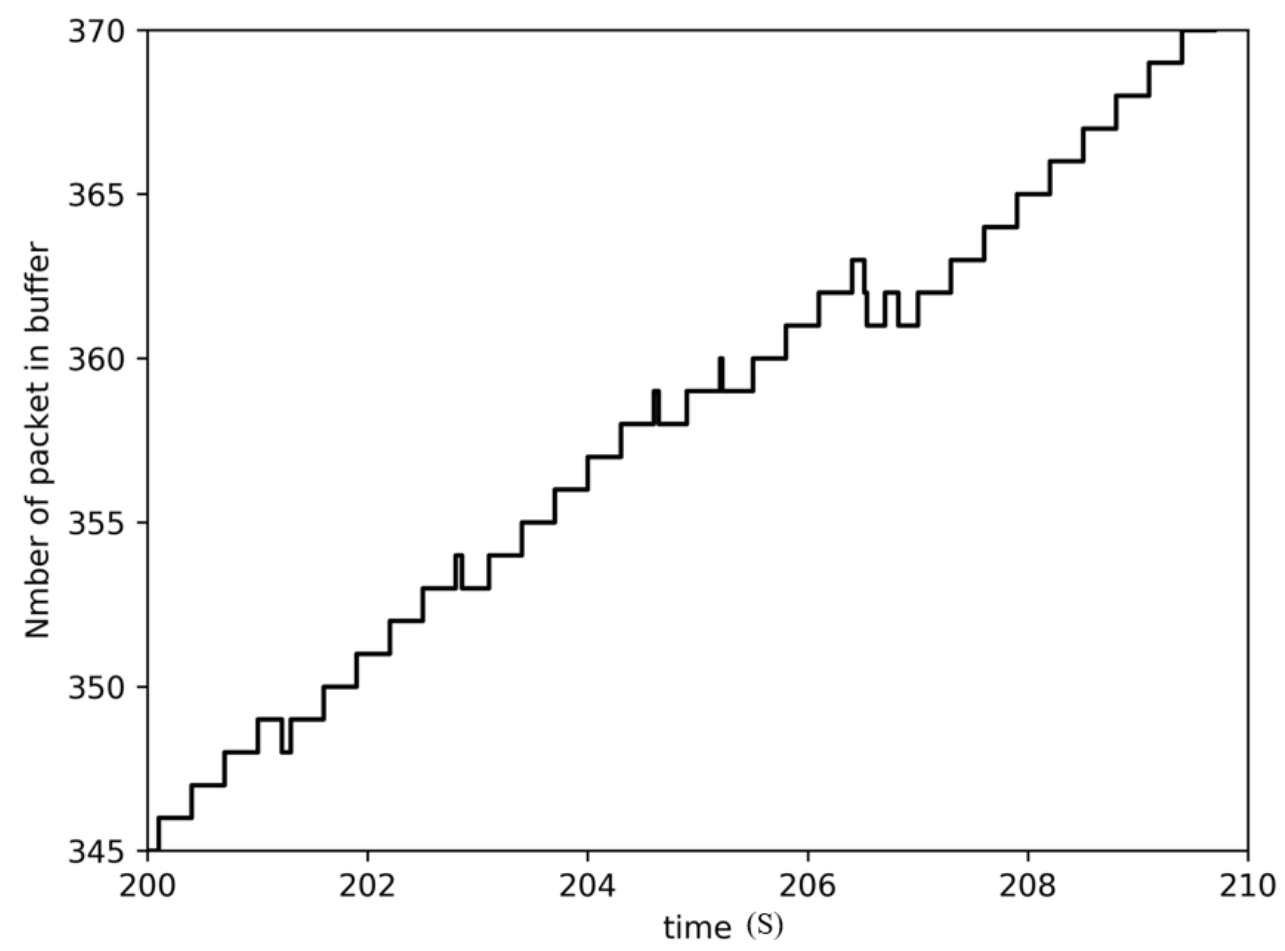

Figure 9. Number of packets in buffer without appropriate DC control.

Figures 10-12 show the PDRs of the IEEE 802.15.4, TMP, and proposed method in scenario 3. The IEEE 802.15.4 and proposed method achieve high PDR. On the other hand, as the data rate of the node increases, the PDR of the TMP decreases. The TMP method has a lower PDR, although it achieves low energy consumption.

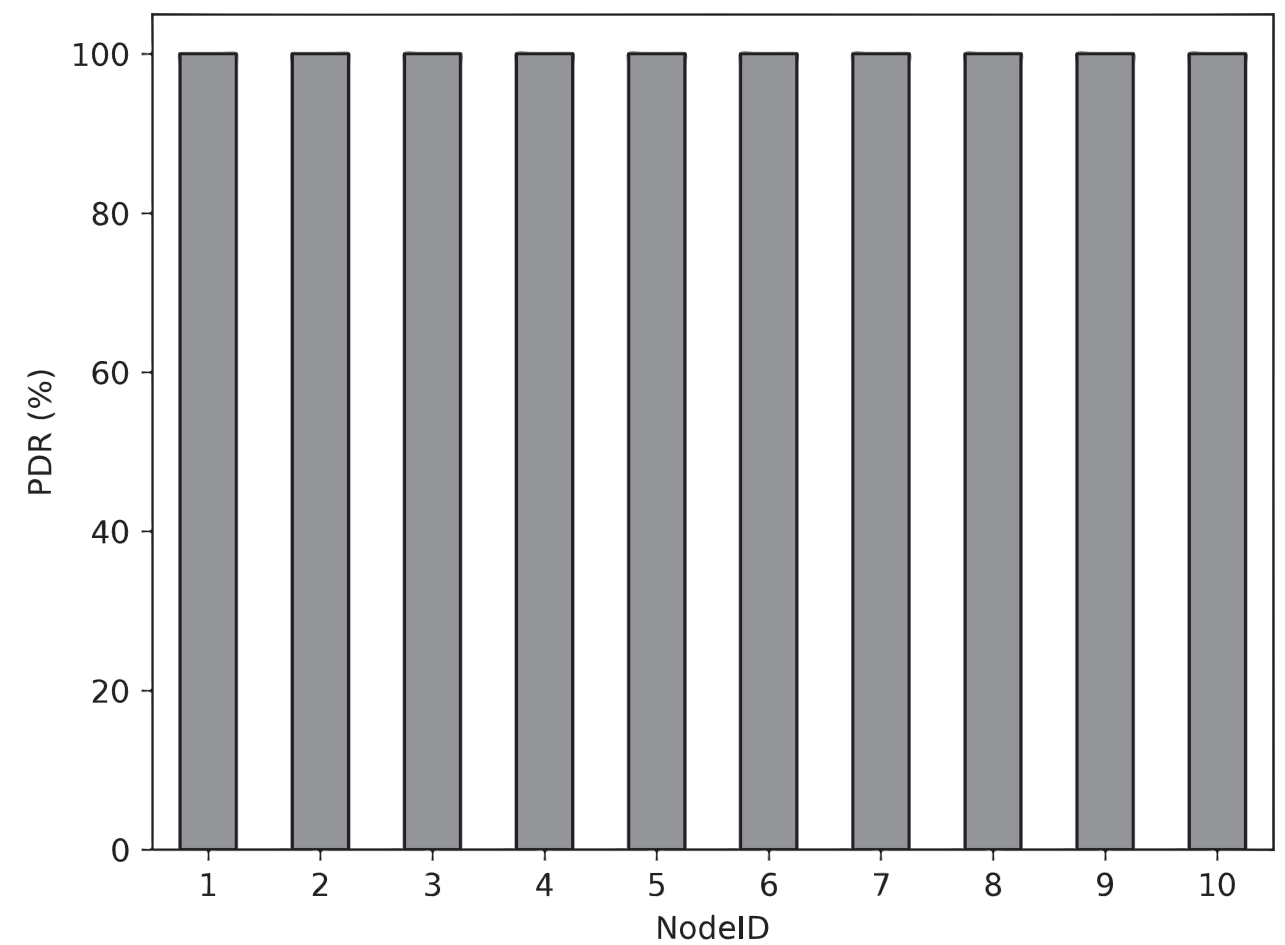

Figure 10. PDR of IEEE 802.15.4 in scenario 3. 


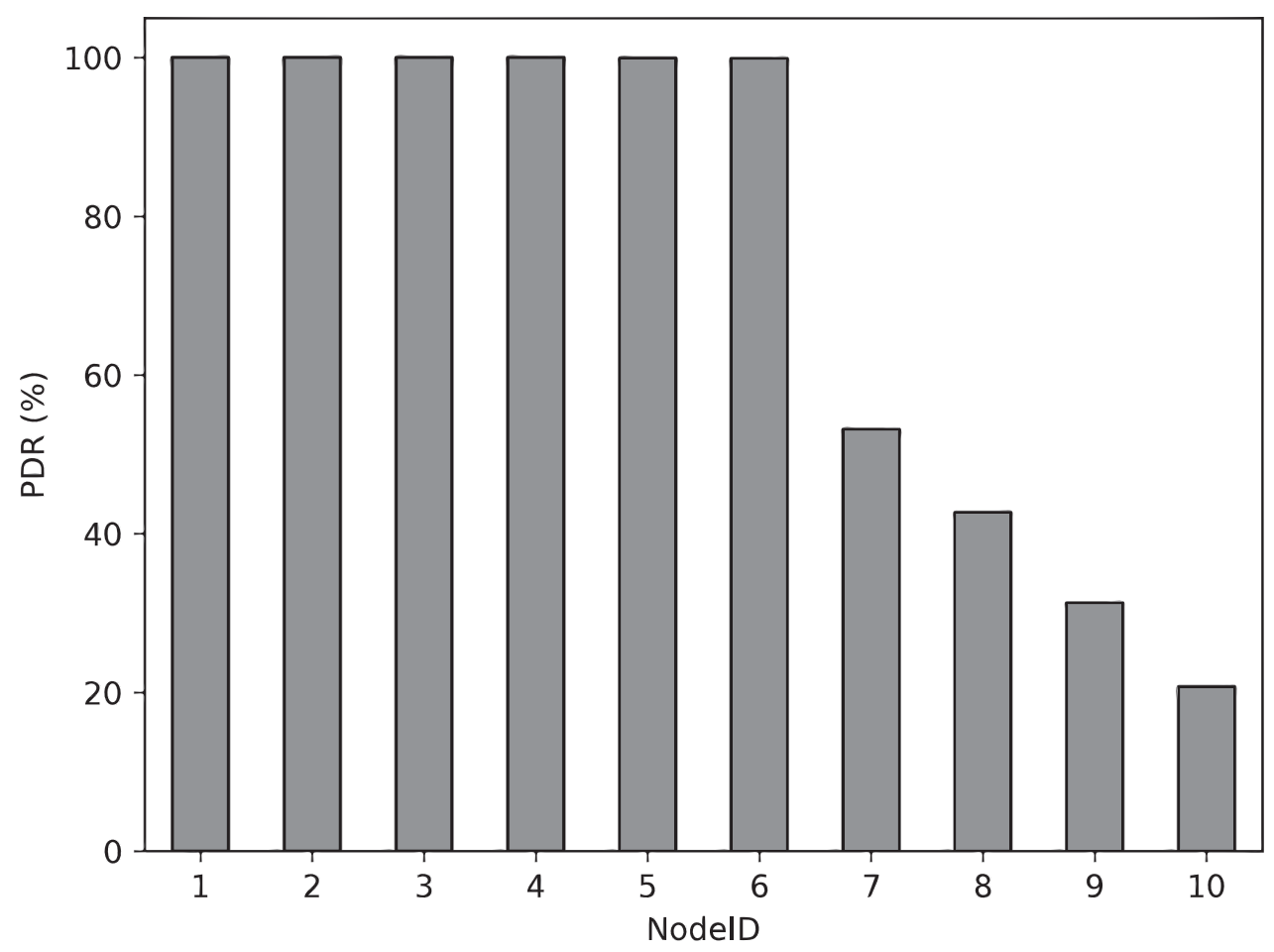

Figure 11. PDR of TMP in scenario 3.

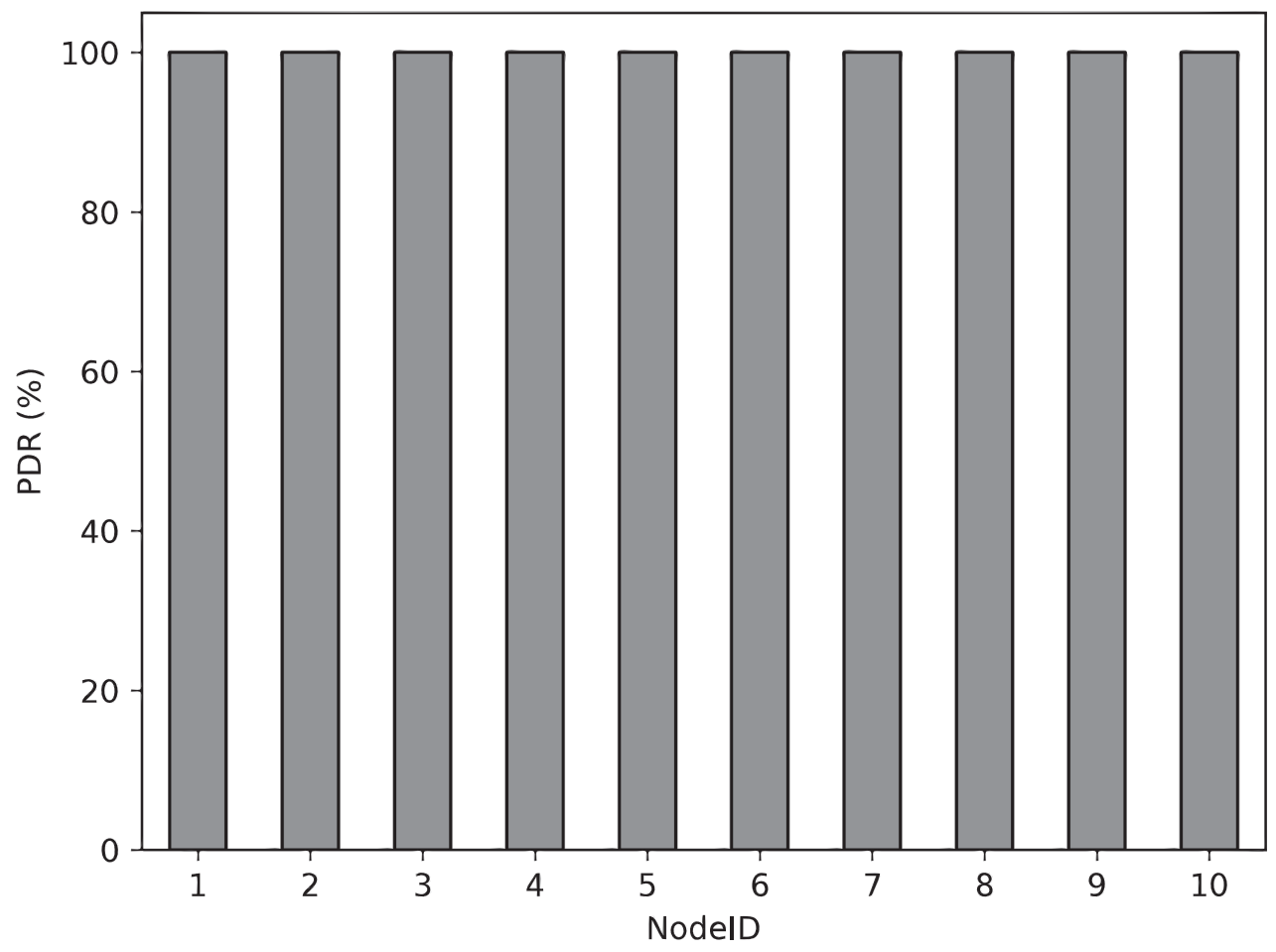

Figure 12. PDR of proposed method in scenario 3.

\subsubsection{Delay Evaluation}

Figure 13 shows the average delay of each scenario and each method. In this study, the delay is defined as the time from the focused packet transmission to arrival at the coordinator. The lower the delivery ratio becomes, the more packet retransmission occurs. If the focused packet is not successfully transmitted within the active period, the packet is 
newly transmitted at the next superframe, which causes extreme delay. In Scenarios 1 and 3 , the delay of TMP increases due to the decrease in PDR.

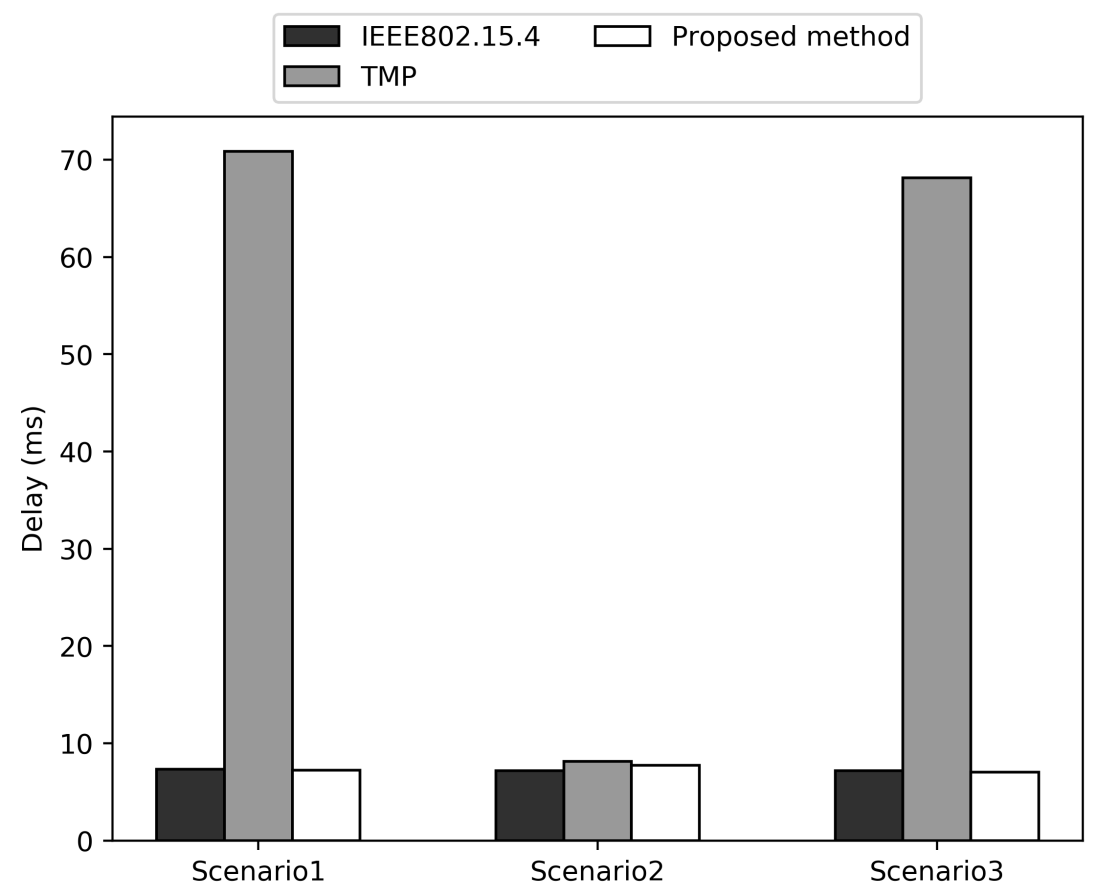

Figure 13. Delay of each scenario.

\subsubsection{Energy Consumption Evaluation}

Figure 14 shows the energy consumption of whole network nodes. The TMP uses low DC, so energy consumption is low. Since IEEE 802.15.4 sets DC regardless of the data rate of the nodes, it wastes the energy by idle listening. The proposed method reduces idle listening by setting appropriate DC for each sub-network while guaranteeing PDR.

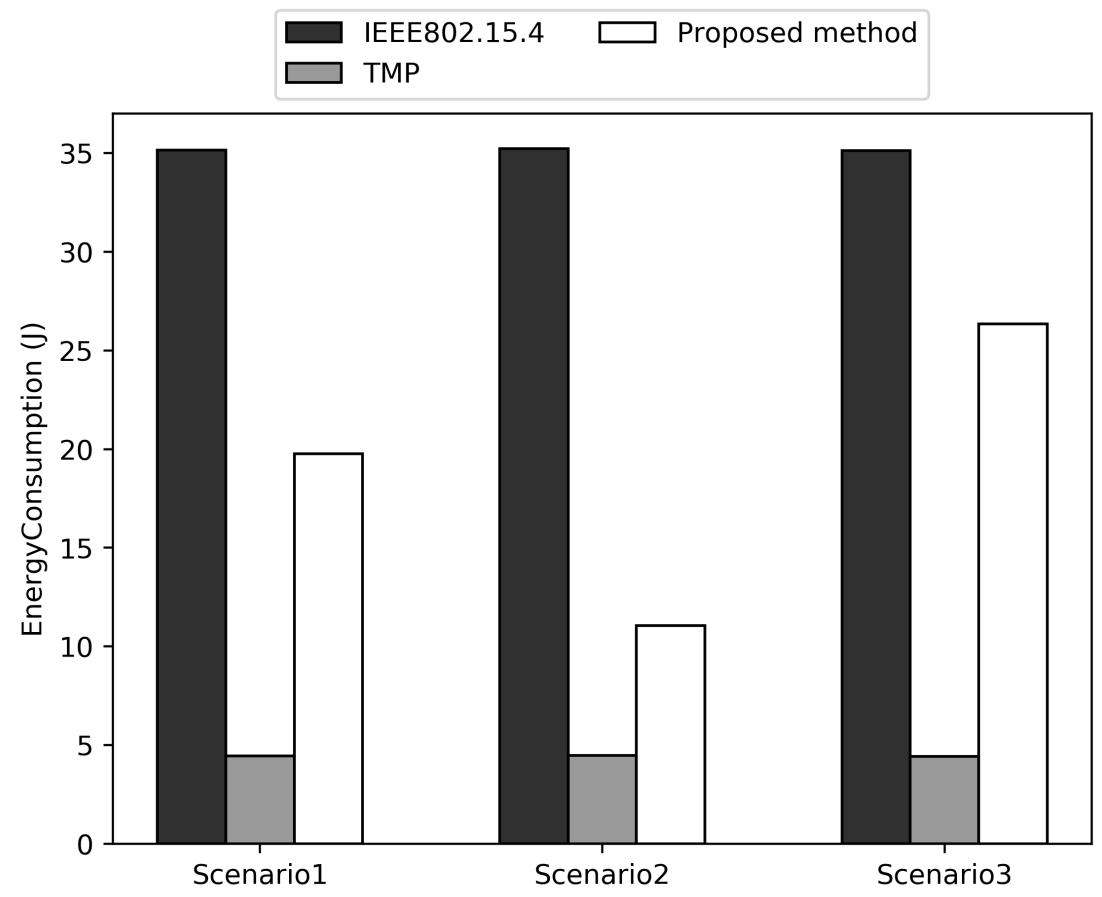

Figure 14. Energy consumption of each scenario. 


\subsubsection{Sensitivity Analysis}

Figures 15-17 shows the sensitivity analysis of the PDR, delay, and energy consumption in scenario 3. The horizontal axis of each graph is the variation of the data rate in the range of $-20 \%$ to $+20 \%$. From Figures $15-17$, the TMP is sensitive to data rate variations regarding PDR and delay, and proposed method is sensitive to data rate variations regarding delay. In addition, these figures illustrate that the variations in data rate do not affect the order of performances.

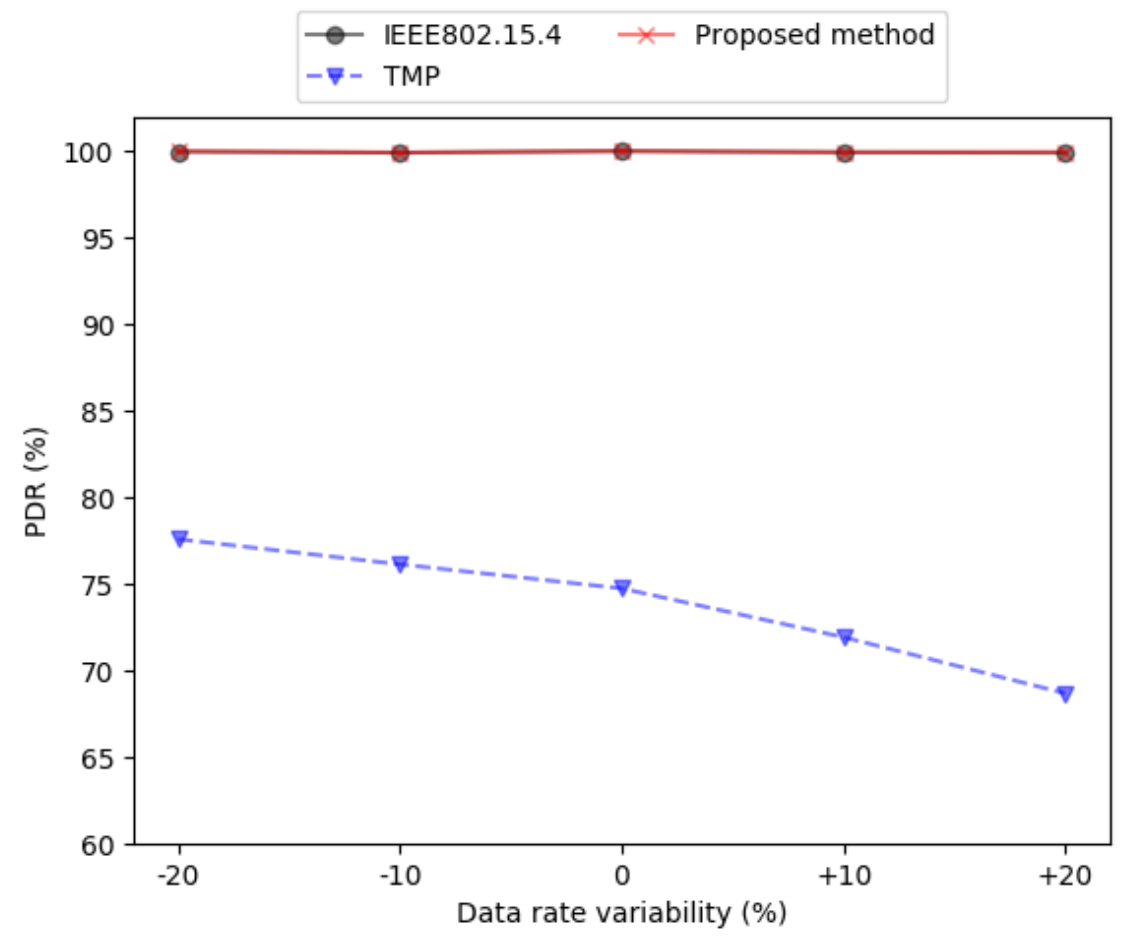

Figure 15. Sensitivity analysis of PDR.

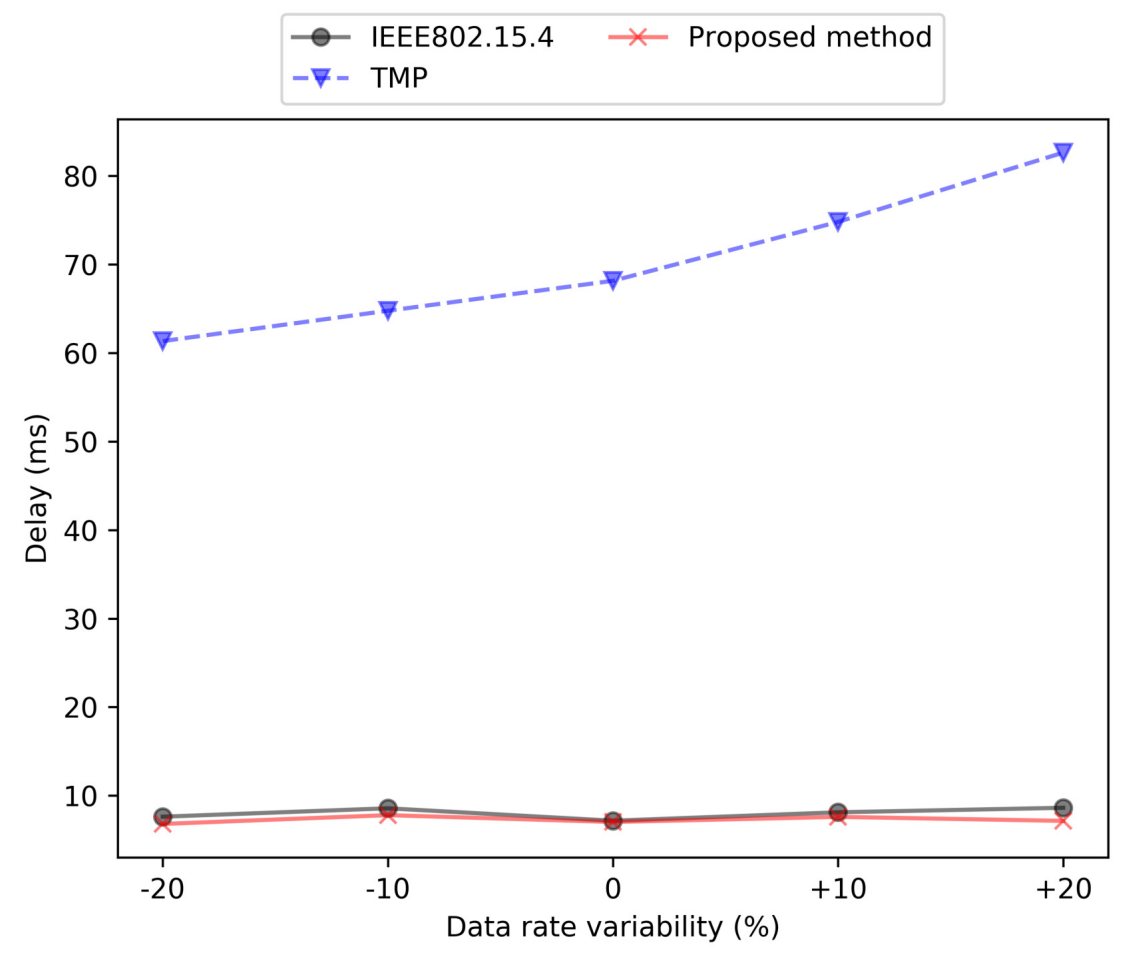

Figure 16. Sensitivity analysis of delay. 


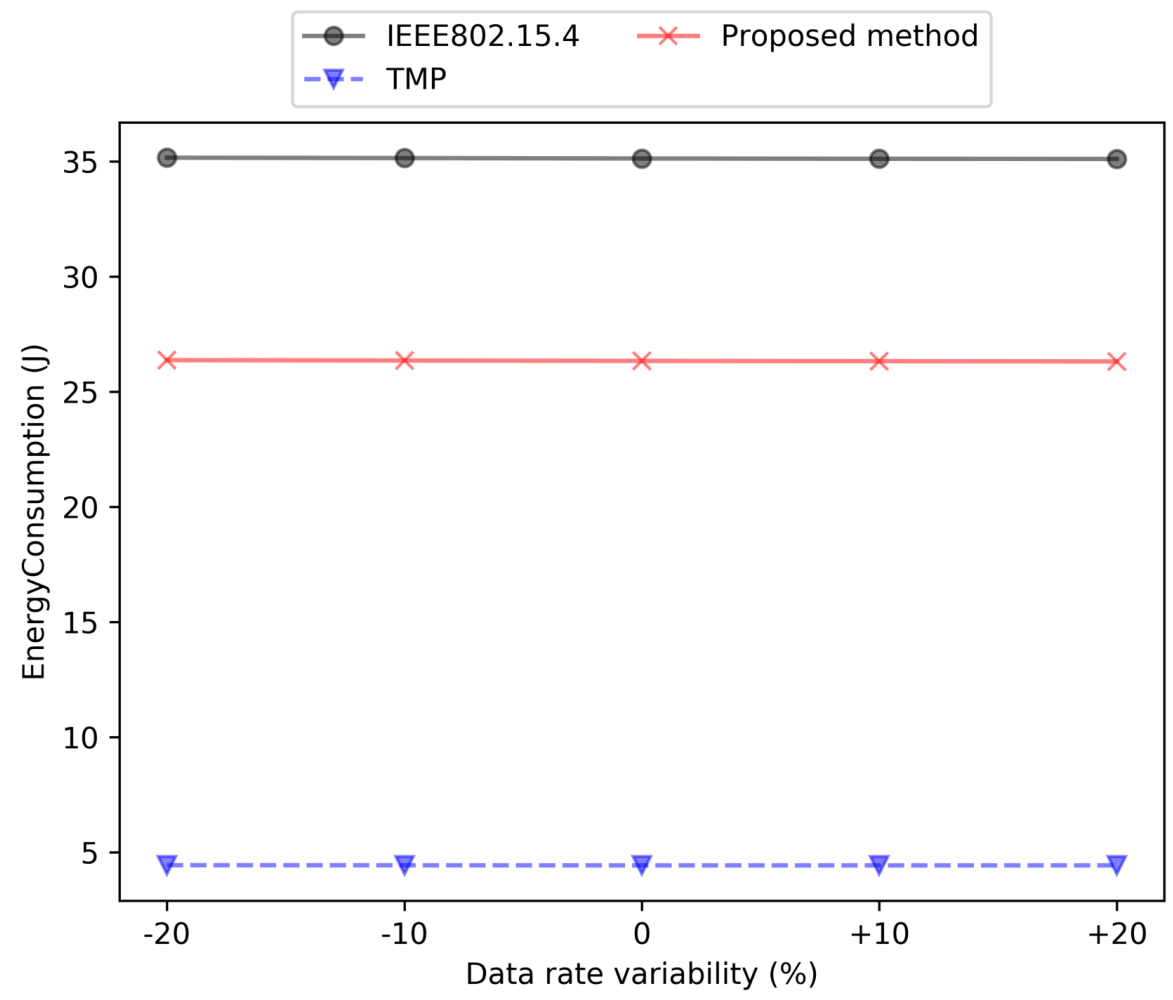

Figure 17. Sensitivity analysis of energy consumption.

\subsection{Summary of Results}

Table 5 summarizes the simulation results of the PDR, delay, and energy consumption of each method. Tables 6-8 show the evaluation criteria for each of Good, Average, and Bad in Table 5 . Table 5 illustrates that the proposed method achieves a reasonable delay and energy consumption while maintaining high PDR.

Table 5. Summary of results.

\begin{tabular}{cccc}
\hline & PDR & Delay & Energy \\
\hline IEEE 802.15.4 & Good & Good & Bad \\
TMP & Average & Bad & Good \\
Proposal & Good & Good & Average \\
\hline
\end{tabular}

Table 6. Evaluation criteria of PDR.

\begin{tabular}{cc}
\hline PDR Range & Grade \\
\hline $90 \%<$ PDR & Good \\
$70 \%<$ PDR $\leq 90 \%$ & Average \\
PDR $\leq 70 \%$ & Bad \\
\hline
\end{tabular}

Table 7. Evaluation criteria of delay.

\begin{tabular}{cc}
\hline Delay Range & Grade \\
\hline delay $<15.36(\mathrm{~ms})$ & Good \\
$15.36(\mathrm{~ms}) \leq$ delay $<30.72(\mathrm{~ms})$ & Average \\
$30.72(\mathrm{~ms}) \leq$ delay & Bad \\
\hline
\end{tabular}


Table 8. Evaluation criteria of energy consumption.

\begin{tabular}{cc}
\hline Energy Consumption Range & Grade \\
\hline energy consumption $<17.5(\mathrm{~J})$ & Good \\
$17.5(\mathrm{~J}) \leq$ energy consumption $<35(\mathrm{~J})$ & Average \\
$35(\mathrm{~J}) \leq$ energy consumption & Bad \\
\hline
\end{tabular}

\section{Conclusions}

This study proposed a DC control method for improving the PDR for IEEE 802.15.4compliant heterogeneous WSNs. The proposed method controls active and sleep periods taking into account the buffer occupancy of nodes with the high data rate. The proposed method tunes the DC so that the buffer occupancy of sensor nodes is less than one. The appropriate DC of each sub-network was used to obtain the high PDR. The simulation results showed that the proposed method achieved a high PDR and low delay not only in the star topology WSN but also in tree topology WSNs.

Author Contributions: N.K. directed the project. N.K. suggested the basic concept of this study. K.T. and N.K. suggested the algorithm demonstrated in this study. K.T. and N.K. designed and obtained results. K.T. and N.K. analyzed the data. All authors contributed the discussions and reviewed the manuscript. All authors have read and agreed to the published version of the manuscript.

Funding: This work was partly supported by The Telecommunications Advancement Foundation, Japan.

Institutional Review Board Statement: Not applicable.

Informed Consent Statement: Not applicable.

Data Availability Statement: The data are available on request from the authors.

Conflicts of Interest: The authors declare no conflict of interest.

\section{References}

1. Hammal, Y.; Ben-Othman, J.; Mokdad, L.; Abdelli, A. Formal Modeling of Greedy Nodes in 802.15.4 WSN. ICT Express 2015, 1, 1-13. [CrossRef]

2. Hadaidian, H.; Yousefi, N.; Kavian, Y.S.; Mahmoudi, A. A markov model for investigating the impact of IEEE802.15.4 MAC layer parameters and number of clusters on the performance of wireless sensor networks. Wirel. Netw. 2019, 25, 4415-4430. [CrossRef]

3. Banu, S.S.; Baskaran, K. Performance Analysis of Immense Preference Data Forwarding (IPDF) Scheme incorporated with Constructive Time Slot Allocation in IEEE 802.15.4 for Wireless Body Area Networks. In Proceedings of the International Conference on Communication and Electronics Systems (ICCES), Coimbatore, India, 7-19 July 2019; pp. 1382-1389.

4. Hayder, A.A.; Kashoash, A.; Hassen, F.; Kharrufa, H.; Kemp, A.H. Analytical modelling of congestion for 6LoWPAN networks. ICT Express 2018, 4, 209-215.

5. Haka, A.; Aleksieva, V.; Valchanov, H. Enhanced Simulation Framework for Visualisation of IEEE 802.15.4 Frame Structure on Beacon Enabled Mode of ZigBee Sensor Network. In Proceedings of the International Conference on Biomedical Innovations and Applications (BIA), Varna, Bulgaria, 24-27 September 2020; pp. 109-112.

6. Hermeto, R.T.; Gallais, A.; Theoleyre, F. Scheduling for IEEE802.15.4-TSCH and slow channel hopping MAC in low power industrial wireless networks: A survey. Comput. Commun. 2017, 14, 84-105. [CrossRef]

7. Luo, C.Y.; Komuro, N.; Takahashi, K.; Tsuboi, T. PPACED TCP: Adynamic Bandwidth Probe TCP with Pacing in Ad Hoc Network. In Proceedings of the IEEE International Symposium on Personal, Indoor and Mobile Radio Communications (PIMRC), Athens, Greece, 3-7 September 2007.

8. Luo, C.Y.; Komuro, N.; Takahashi, K.; Tsuboi, T.; Ueda, H. Enhancing QoS Provision by Priority Scheduling with Interference Drop Scheme in Multi-hop Ad Hoc Networks. In Proceedings of the IEEE Global Communications Conference (GLOBECOM), Orleans, LA, USA, 30 November-4 December 2008.

9. Komuro, N.; Habuchi, H.; Tsuboi, T. Nonorthogonal CSK/CDMA with Received-Power Adaptive Access Control Scheme. IEICE Trans. Fundam. Electron. Commun. Comput. Sci. 2008, 91, 2779-2786. [CrossRef]

10. Tuan, D.T.; Sakata, S.; Komuro, N. Priority and admission control for assuring quality of I2V emergency services in VANETs integrated with Wireless LAN Mesh Networks. In Proceedings of the International Conference on Communications and Electronics (ICCE), Hue, Vietnam, 1-3 August 2012.

11. Ma, J.; Sekiya, H.; Nagasaki, A.; Komuro, N.; Sakata, S. MAC Protocol for Ad Hoc Networks Using Smart Antennas for Mitigating Hidden and Deafness Problems. IEICE Trans. Commun. 2012, 95, 3545-3555. [CrossRef] 
12. Davoli, L.; Antonini, M.; Ferrari, G. DiRPL: A RPL-Based Resource and Service Discovery Algorithm for 6LoWPANs. Appl. Sci. 2019, 9, 33. [CrossRef]

13. Takizawa, S.; Komuro, N.; Sakata, S. Routing Control Scheme Prolonging Network Lifetime in a 6LoWPAN WSN with Powersupplied and Battery-powered Nodes. In Proceedings of the IEEE Consumer Communications and Networking Conference (CCNC), Las Vegas, NV, USA, 14-17 January 2012.

14. Wang, Y.; Yang, W.; Han, R.; Xu, L.; Zhao, H. An Analytical Framework for the IEEE 802.15.4 MAC Layer Protocol under Periodic Traffic. Sensors 2020, 20, 3350. [CrossRef] [PubMed]

15. Chu, Z.; Sun, W.; Wang, J. Research on MAC Layer Communication Performance Model of Wireless Sensor Networks for Intelligent Transportation. In Proceedings of the International Conference on Automation and Computing (ICAC), Colchester, UK, 7-8 September 2016.

16. Bouazzi, I.; Bhar, J.; Atri, M. Priority-based queuing and transmission rate management using a fuzzy logic controller in WSNs. ICT Express 2017, 3, 101-105. [CrossRef]

17. Qiu, T.; Chen, N.; Li, K.; Atiquzzaman, M.; Zhao, W. How Can Heterogeneous Internet of Things Build Our Future: A Survey. IEEE Commun. Surv. Tutor. 2018, 20, 2011-2027. [CrossRef]

18. Akbar, M.S.; Yu, H.; Cang, S. TMP: Tele-Medicine Protocol for Slotted 802.15.4 With Duty-Cycle Optimization in Wireless Body Area Sensor Networks. IEEE Sens. J. 2017, 17, 1925-1936. [CrossRef]

19. IEEE Standards Association. IEEE Standard for Local and Metropolitan Area Networks Part 15.4: Low-Rate Wireless Personal Area Networks (LR-WPANs). Available online: http:/ / ecee.colorado.edu/ liue/teaching/comm-standards/2015S-zigbee/802.15 .4-2011.pdf (accessed on 10 December 2020).

20. Xiao, Z.; Zhou, J.; Yan, J.; He, C.; Jiang, L.; Trigoni, N. Performance evaluation of IEEE 802.15.4 with real time queueing analysis. Ad Hoc Netw. 2018, 73, 80-94. [CrossRef]

21. Elsts, A. TSCH-Sim: Scaling Up Simulations of TSCH and 6TiSCH Networks. Sensors 2020, 20, 5663. [CrossRef] [PubMed]

22. Karalis, A.; Zorbas, D.; Douligeris, C. Collision-Free Advertisement Scheduling for IEEE 802.15.4-TSCH Networks. Sensors 2019, 19, 1789. [CrossRef] [PubMed]

23. Sekiya, H.; Tsuchiya, Y.; Komuro, N.; Sakata, S. Analytical Expressions of Maximum Throughput for Long-Frame Communications in One-way String Wireless Multihop Networks. Wirel. Pers. Commun. 2011, 60, 29-41. [CrossRef]

24. Sanada, K.; Komuro, N.; Sekiya, H. End-to-end throughput and delay analysis for IEEE 802.11 string topology multi-hop network using markov-chain model. In Proceedings of the IEEE International Symposium on Personal, Indoor and Mobile Radio Communications (PIMRC), Hong Kong, China, 30 August-2 September 2015.

25. Sanada, K.; Shi, J.; Komuro, N.; Sekiya, H. End-to-End Delay Analysis for IEEE 802.11 String-Topology Multi-hop Networks. IEICE Trans. Commun. 2015, 95, 1284-1293. [CrossRef]

26. Wan, Y.; Sanada, K.; Komuro, N.; Motoyoshi, G.; Yamagaki, N.; Shioda, S.; Murase, T.; Sekiya, H. Throughput analysis of WLANs in saturation and non-saturation heterogeneous conditions with airtime concept. IEICE Trans. Commun. 2016, 95, 2289-2296. [CrossRef]

27. The Network Simulator - ns-2. Available online: https://www.isi.edu/nsnam/ns (accessed on 15 November 2020).

28. Ramachandran, I.; Das, A.K.; Roy, S. Analysis of the contention access period of IEEE 802.15.4 MAC. ACM Trans. Sens. Netw. 2007, 3, 1706-1718. [CrossRef] 